\title{
An Equilibrium Chance-Constrained Multiobjective Programming Model with Birandom Parameters and Its Application to Inventory Problem
}

\author{
Zhimiao Tao ${ }^{1}$ and Jiuping $\mathrm{Xu}^{1,2}$ \\ ${ }^{1}$ Uncertainty Decision-Making Laboratory, Sichuan University, Chengdu 610064, China \\ ${ }^{2}$ State Key Laboratory of Hydraulics and Mountain River Engineering, Sichuan University, Chengdu 610064, China \\ Correspondence should be addressed to Jiuping Xu; xujiuping@scu.edu.cn
}

Received 14 May 2013; Revised 25 July 2013; Accepted 25 July 2013

Academic Editor: Wei-Shih Du

Copyright ( $\odot 2013$ Z. Tao and J. Xu. This is an open access article distributed under the Creative Commons Attribution License, which permits unrestricted use, distribution, and reproduction in any medium, provided the original work is properly cited.

An equilibrium chance-constrained multiobjective programming model with birandom parameters is proposed. A type of linear model is converted into its crisp equivalent model. Then a birandom simulation technique is developed to tackle the general birandom objective functions and birandom constraints. By embedding the birandom simulation technique, a modified genetic algorithm is designed to solve the equilibrium chance-constrained multiobjective programming model. We apply the proposed model and algorithm to a real-world inventory problem and show the effectiveness of the model and the solution method.

\section{Introduction}

As a key issue in supply chain management, inventory management plays an important role in controlling operation cost and improving management efficiency. As a result, inventory problems have attracted many researchers from various fields. Classical inventory problem research discussed single-item cases [1-5]. However, multiitem inventory problems are more common in the real-world. In fact, for many enterprises, especially supermarkets, it is necessary to prepare hundreds of goods to meet the needs of different consumers. Therefore, multiitem inventory problem research has become more attractive for researchers [6-9].

Apart from the multiitem inventory, uncertainty is another characteristic of inventory problems. For example, the price of a product usually varies due to the market fluctuation. One kind of common uncertainty is randomness. Many researchers have studied random inventory models. Baker and Ehrhardt analyzed a periodic-review, random-demand inventory model under the assumption that replenishment quantities are random fractions of the amounts ordered [10]. Bera et al. dealt with a multiitem mixture inventory model in which both demand and lead time are random [8]. Fuzziness is another type of uncertainty in inventory problems. Wang et al. [11] developed a novel joint replenishment problem model with fuzzy minor replenishment cost and fuzzy inventory holding cost. Roy et al. [12] considered an inventory model for a deteriorating item (seasonal product) with linearly displayed stock dependent demand in an imprecise environment (involving both fuzzy and random parameters) under inflation and the time value of money. M. K. Maiti and M. Maiti [13] proposed a multiitem inventory model with advertising costs, price, and displayed inventory leveldependent demand in a fuzzy environment (purchase cost, investment amount, and storehouse capacity were considered imprecise). Besides these, twofold uncertain inventory models have also been studied as more complex; imprecise information is considered. Dutta et al. [14] presented a singleperiod inventory problem in an imprecise and uncertain mixed environment, in which the demand is assumed as a fuzzy random variable. $\mathrm{Xu}$ and Zhao $[15,16]$ formulated inventory models under fuzzy rough environments.

However, an inventory model with twofold random phenomenon is seldom discussed. In reality, inventory problems 
in the real-world may be subjected to twofold randomness with incomplete or uncertain information. Consequently, developing an inventory strategy in a more complete stochastic environment takes place. As a case in point, it is widely accepted that the price of a product is a normal distributed variable, denoted by $N\left(\mu, \sigma^{2}\right)$ from the viewpoint of probability theory, but the values of $\mu$ and $\sigma$ may be still uncertain variables. If there is statistical information about $\mu$ and $\sigma$, it is possible to specify realistic distributions for $\mu$ and $\sigma$ by utilizing statistical methods. When the values of $\mu$ and $\sigma$ are provided as random variables, the price then is not a conventional random variable but a so-called birandom variable. A birandom variable, which plays a role analogous to a random variable in probability theory, is appropriate to describe this kind of twofold randomness. In this paper, we discuss a class of multiobjective programming models with birandom parameters. Chance-constrained programming proposed by Charnes and Cooper [17] is an effective technique to deal with uncertain optimization problem [18]. By using the equilibrium chance-constrained programming technique, the initial model is meaningful mathematically.

The rest of the paper is organized as follows. Section 2 develops a general equilibrium chance-constrained programming model with birandom parameters. A linear model is converted into its crisp equivalent model. In order solve a general model, the birandom simulation-based genetic algorithm is deigned in Section 3. Section 4 applies the theoretical results into a real-world inventory problem. Further discussions in Section 5 illustrate the effectiveness of the proposed model and algorithm. Finally, concluding remarks are outlined in Section 6.

\section{Equilibrium Chance-Constrained Multiobjective Programming Model with Birandom Parameters}

In order construct a general equilibrium chance-constrained multiobjective programming model with birandom parameters, we first state some basic concepts and theorems on birandom theory which is presented firstly.

2.1. Birandom Variable and Equilibrium Chance. Birandom variable, which is proposed by Peng and Liu [19], is a mathematical tool to describe twofold random phenomena. An $n$ dimensional birandom vector $\xi$ is a map from the probability space $(\Omega, \mathscr{A}, \operatorname{Pr})$ to a collection of $n$-dimensional random vectors such that $\operatorname{Pr}\{\xi(\omega) \in \mathscr{B}\}$ is a measurable function with respect to $\omega$ for any Borel set $\mathscr{B}$ of the real space $\mathscr{R}^{n}$. In particular, $\xi$ is called a birandom variable as $n=1$.

Example 1. A birandom variable $\xi$ is said to be uniform, if for each $\omega, \xi(\omega)$ is a random variable with uniform distribution, denoted by $\mathscr{U}[a(\omega), b(\omega)]$, where $a(\omega)$ and $b(\omega)$ are random variables defined on the probability space $(\Omega, \mathscr{A}, \operatorname{Pr})$.

Example 2. A birandom variable $\xi$ is said to be normal, if for each $\omega, \xi(\omega)$ is a random variable with normal distribution,

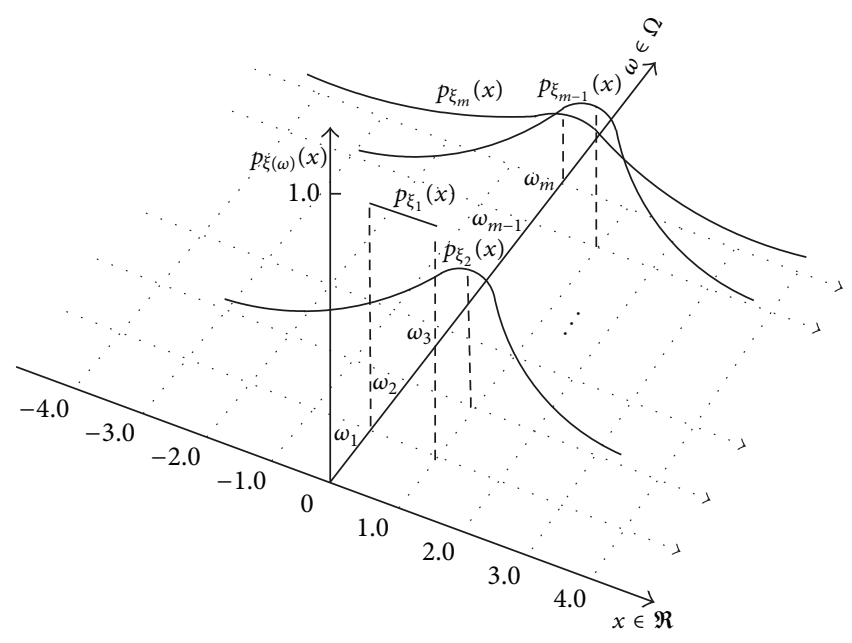

Figure 1: A birandom variable.

denoted by $\mathcal{N}\left(\mu(\omega), \sigma^{2}(\omega)\right)$, where $\mu(\omega)$ and $\sigma(\omega)$ are random variables defined on the probability space $(\Omega, \mathscr{A}, \operatorname{Pr})$.

Example 3. A birandom variable $\xi$ is said to be exponential, if for each $\omega, \xi(\omega)$ is a random variable with exponential distribution, denoted by $\exp (\lambda(\omega))$, where $\lambda(\omega)$ are random variables defined on the probability space $(\Omega, \mathscr{A}, \operatorname{Pr})$.

Example 4. Let $(\Omega, \mathscr{A}, \operatorname{Pr})$ be a probability space. If $\Omega=$ $\left\{\omega_{1}, \omega_{2}, \ldots, \omega_{n}\right\}$,

$$
\xi(\omega)= \begin{cases}\xi_{1}, & \omega=\omega_{1} \\ \xi_{2}, & \omega=\omega_{2} \\ \vdots, & \vdots \\ \xi_{n}, & \omega=\omega_{n},\end{cases}
$$

where $\xi_{1}, \xi_{2}, \ldots, \xi_{n}$ are random variables with density function $p_{1}(x), p_{1}(x), \ldots, p_{n}(x)$, respectively. Birandom variable $\xi$ is illustrated by Figure 1 .

By birandom event we mean that $g(\xi) \leq 0$. In order to compare the degrees of occurrence of two birandom events, quantitative measures of the chance of a birandom event are necessary. In the literature, the first attempt to develop the definition of the chance of a birandom event is primitive chance, which is a function from $[0,1]$ to $[0,1]$.

Definition 5 (see [19]). Let $\xi=\left(\xi_{1}, \xi_{2}, \ldots \xi_{n}\right)$ be a birandom vector defined on $\Omega$, and $g: \mathfrak{R}^{n} \mapsto \Re$ is Borel measurable function. Then the primitive chance of a birandom event characterized by $g(\xi) \leq 0$ is a function from $[0,1]$ to $[0,1]$, defined as

$$
\begin{aligned}
\operatorname{Ch} & \{g(\xi) \leq 0\}(\alpha) \\
& =\sup _{\alpha \in[0,1]}\{\beta \mid \operatorname{Pr}\{\omega \in \Omega \mid \operatorname{Pr}\{g(\xi(\omega)) \leq 0\} \geq \beta\} \geq \alpha\},
\end{aligned}
$$


where $\alpha$ is a prescribed probability level. The value of primitive chance at $\alpha$ is called $\alpha$-chance.

Remark 6. It should be noted that the symbol Pr appears twice in the right side of (2). In fact, they represent different meanings. In other words, the overloading allows us to use the same symbol Pr for different probability measures, because we can deduce the exact meaning in the context.

In the case of fuzzy random programming problems, the equilibrium chance of a fuzzy random event was introduced to measure the degree of the occurrence of a fuzzy random event [20]. Rather than a function, the equilibrium chance is a scalar value, like the probability of a random event and the possibility of a fuzzy event. Thus it is easy for the decision maker to rank the decisions via equilibrium chance using the natural order of real numbers, rather than requiring a preference order from the decision maker. Motivated by the idea, we introduce the equilibrium chance of a birandom event as follows.

Definition 7. Let $\xi=\left(\xi_{1}, \xi_{2}, \ldots, \xi_{n}\right)$ be a birandom vector, and $g: \mathfrak{R}^{n} \mapsto \mathfrak{R}$ is a Borel measurable function. Then the equilibrium chance of birandom event $g(\xi) \leq 0$ is defined as

$$
\begin{aligned}
\mathrm{Ch}^{e} & \{g(\xi) \leq 0\} \\
& =\sup _{\alpha \in[0,1]}\{\alpha \wedge \operatorname{Pr}\{\omega \in \Omega \mid \operatorname{Pr}\{g(\xi(\omega)) \leq 0\} \geq \alpha\} .
\end{aligned}
$$

Remark 8. Peng and Liu [19] introduced the equilibrium chance of a birandom event as

$$
\mathrm{Ch}^{e}\{g(\xi) \leq 0\}=\sup _{\alpha \in[0,1]}\{\alpha \mid \operatorname{Ch}\{g(\xi) \leq 0\}(\alpha) \geq \alpha\},
$$

where $\mathrm{Ch}$ is the primitive chance. It is easy to verify the forms (3) and (4) are equivalent.

Remark 9. If $\xi$ degenerates to a random vector, $\operatorname{Pr}\{\omega \in \Omega \mid$ $\operatorname{Pr}\{g(\xi(\omega)) \leq 0\} \geq \alpha\}$ implies $\operatorname{Pr}\{g(\xi(\omega)) \leq 0\} \geq \alpha$, and then (3) is equivalent to $\operatorname{Pr}\{g(\xi(\omega)) \leq 0\}$, which is the probability measure.

For any $\alpha \in[0,1]$, denote

$$
\begin{gathered}
F(\alpha)=\operatorname{Pr}\{\omega \in \Omega \mid \operatorname{Pr}\{g(\xi(\omega)) \leq 0\} \geq \alpha\}, \\
F(\alpha+)=\operatorname{Pr}\{\omega \in \Omega \mid \operatorname{Pr}\{g(\xi(\omega)) \leq 0\}>\alpha\} .
\end{gathered}
$$

It follows from the definition of birandom variable that $\operatorname{Pr}\{(\xi(\omega)) \leq 0\}$ is a random variable. Then $F(\alpha)$ is a leftcontinuous and nonincreasing function with respect to $\alpha$, and for any given $\alpha, F(\alpha)$ represents the following random event occurring:

$$
\{\omega \in \Omega \mid \operatorname{Pr}\{g(\xi(\omega)) \leq 0\} \geq \alpha\} .
$$

Therefore, the equilibrium chances defined above measure the twofold maximum probability simultaneously.

Generally, if

$$
F(\alpha) \leq \alpha \leq F(\alpha+),
$$

then $\alpha$ is just the value of the equilibrium chance. As $\operatorname{Pr}\{g(\xi(\omega)) \leq 0\}$ is a continuous random variable, the left side and right side of relation (7) are equal. Thus the equilibrium chance is available at the fixed point of $F$; that is, $F(\alpha)=\alpha$.

It follows from the definitions of equilibrium chance and primitive chance of a birandom event that they have the following differences.

(i) The equilibrium chance of a birandom chance of a birandom event is a scalar value, just like the probability of a random event and the possibility of a fuzzy event, while the primitive chance of a birandom event is a function from $[0,1]$ to $[0,1]$.

(ii) The equilibrium chance measures the twofold probabilities at the same time, while the value of the primitive chance at $\alpha$ measures the single maximum probability of a birandom event occurring under the given value of the other probability.

However, the connections of the equilibrium chance and the primitive chance can be summarized as follows.

(i) Equilibrium chance (3) can be represented as

$$
\operatorname{Ch}^{e}\{g(\xi) \leq 0\}=\sup _{\alpha \in[0,1]}\left\{\alpha \wedge \operatorname{Ch}\left\{g_{r}(\xi(\omega)) \leq 0\right\}(\alpha)\right\},
$$

where $\operatorname{Ch}\{g(\xi(\omega)) \leq 0\}(\alpha)$ is the value of primitive chance at $\alpha$.

(ii) The primitive chance of a birandom event is the pseudo-inverse function of the function $F(\alpha)$.

In order to generate the notation of the equilibrium chance of a birandom event defined by (3), we give another equivalent form as follows:

$$
\begin{aligned}
& \operatorname{Ch}^{e}\{g(\xi) \leq 0\} \\
& =\sup _{(\alpha, \beta) \in[0,1]^{2}}\{\alpha \wedge \beta \mid \operatorname{Pr}\{\omega \in \Omega \mid \operatorname{Pr}\{g(\xi(\omega)) \leq 0\} \\
& \geq \alpha\} \geq \beta\},
\end{aligned}
$$

where the parameters $\alpha$ and $\beta$ represent two kinds of probability. It is easy to see from (3) that we use the $\min / \max$ operator, which is a special triangular norm, to define the equilibrium chance of a birandom event. In fact, by using a common triangular norm $T$, the equilibrium chance can be extended as

$$
\begin{aligned}
& \operatorname{Ch}_{T}^{e}\{g(\xi) \leq 0\} \\
& =\sup _{(\alpha, \beta) \in[0,1]^{2}}\{T(\alpha, \beta) \mid \\
& \left.\quad \operatorname{Pr}\left\{\omega \in \Omega \mid \operatorname{Pr}\left\{f_{j}(\xi(\omega)) \leq 0\right\} \geq \alpha\right\} \geq \beta\right\},
\end{aligned}
$$

where $T:[0,1] \mapsto[0,1]$ is a triangular norm. In practice, we may also use various triangular norms such as $T_{1}(\alpha, \beta)=\alpha \beta$, $T_{2}(\alpha, \beta)=\alpha \beta /[1+(1-\alpha)(1-\beta)], T_{3}(\alpha, \beta)=\alpha \beta /(\alpha+\beta-\alpha \beta)$, 
or $T_{4}=\max \{0, \alpha+\beta+1\}$ according to a decision maker's philosophy of modeling uncertainty.

The following theorem implies a basic property of the equilibrium chance.

Theorem 10. Let $\xi=\left(\xi_{1}, \xi_{2}, \ldots, \xi_{n}\right)$ be a birandom vector, and let $g: \mathfrak{R}^{n} \mapsto \mathfrak{R}$ be Borel measurable function, and $\alpha \in[0,1]$. Then

$$
\begin{aligned}
\mathrm{Ch}^{e} & \{g(\xi) \leq 0\} \\
& \geq \alpha \Longleftrightarrow \operatorname{Pr}\{\omega \in \Omega \mid \operatorname{Pr}\{g(\xi(\omega)) \leq 0\} \geq \alpha\} \geq \alpha .
\end{aligned}
$$

Proof. " $\Rightarrow$ ” Assume that $\mathrm{Ch}^{e}\{g(\xi) \leq 0\} \geq \alpha$. It follows from the definition of $\mathrm{Ch}^{e}\{\cdot\}$ that there exists a real number $\alpha_{0} \in$ $[0,1]$ such that

$$
\alpha_{0} \wedge \operatorname{Pr}\left\{\omega \in \Omega \mid \operatorname{Pr}\{g(\xi(\omega)) \leq 0\} \geq \alpha_{0}\right\} \geq \alpha .
$$

Thus we have

$$
\begin{gathered}
\alpha_{0} \geq \alpha, \\
\operatorname{Pr}\{g(\xi(\omega)) \leq 0\} \geq \alpha_{0} \geq \alpha .
\end{gathered}
$$

So we get that

$$
\begin{aligned}
& \{\omega \in \Omega \mid \operatorname{Pr}\{g(\xi(\omega)) \leq 0\} \geq \alpha\} \\
& \quad \supseteq\left\{\omega \in \Omega \mid \operatorname{Pr}\{g(\xi(\omega)) \leq 0\} \geq \alpha_{0}\right\} .
\end{aligned}
$$

It follows that

$$
\begin{aligned}
& \operatorname{Pr}\{\omega \in \Omega\{g(\xi(\omega)) \leq 0\} \geq \alpha\} \\
& \quad \geq \operatorname{Pr}\left\{\omega \in \Omega\{g(\xi(\omega)) \leq 0\} \geq \alpha_{0}\right\} \geq \alpha .
\end{aligned}
$$

“ $\Leftarrow$ " If $\operatorname{Pr}\{\omega \in \Omega \mid \operatorname{Pr}\{g(\xi(\omega)) \leq 0\} \geq \alpha\} \geq \alpha$, then we have

$$
\begin{aligned}
\alpha & =\alpha \wedge \operatorname{Pr}\{\omega \in \Omega \mid \operatorname{Pr}\{g(\xi(\omega)) \leq 0\}\} \\
& \leq \sup _{\beta \in[0,1]}\{\beta \wedge \operatorname{Pr}\{\omega \in \Omega \mid \operatorname{Pr}\{g(\xi(\omega)) \leq 0\} \geq \beta\}\} \\
& =\operatorname{Ch}^{e}\{g(\xi(\omega)) \leq 0\} .
\end{aligned}
$$

The theorem is proved.

The independence of birandom variables is defined as follows.

Definition 11. Birandom variables $\xi_{1}, \xi_{2}, \ldots, \xi_{n}$, which are defined on the probability space $(\Omega, \mathscr{A}, \operatorname{Pr})$, are said to be independent if and only if $\xi_{1}(\omega), \xi_{2}(\omega), \ldots, \xi_{n}(\omega)$ are independent random variables for all $\omega \in \Omega$.

2.2. Model Formulation. A general equilibrium chance-constrained multiobjective programming model with birandom parameters is formulated as

$$
\begin{array}{ll}
\max & \quad\left\{\bar{f}_{1}, \bar{f}_{2}, \ldots, \bar{f}_{m}\right\} \\
\text { s.t. } \quad & \operatorname{Ch}^{e}\left\{f_{i}(x, \xi) \geq \bar{f}_{i}\right\} \geq \alpha_{i}, \quad i=1,2, \ldots, m, \\
& \operatorname{Ch}^{e}\left\{g_{r}(x, \xi) \leq 0\right\} \geq \beta_{r}, \quad r=1,2, \ldots, p, \\
& x \in D,
\end{array}
$$

where $x$ is an $n$-ary decision vector, $\xi m$-ary is a birandom vector, $D$ is a fixed set that is usually determined by a finite number of inequalities involving functions of $x, f_{i}$ and $g_{r}$ are $(m+n)$-ary real-valued continuous functions, and $\alpha_{i}$ and $\beta_{r}$ are predetermined confidence levels, $i=1,2, \ldots, m, r=$ $1,2, \ldots, p$.

It follows from Remark 9 that if $\xi$ degenerates to random vector, then model (17) degenerates to

$$
\begin{array}{ll}
\max & \left\{\bar{f}_{1}, \bar{f}_{2}, \ldots, \bar{f}_{m}\right\} \\
\text { s.t. } & \operatorname{Pr}\left\{f_{i}(x, \xi) \geq \bar{f}_{i}\right\} \geq \alpha_{i}, \quad i=1,2, \ldots, m, \\
& \operatorname{Pr}\left\{g_{r}(x, \xi) \leq 0\right\} \geq \beta_{r}, \quad r=1,2, \ldots, p, \\
& x \in D,
\end{array}
$$

which is a stochastic chance-constrained programming model.

Definition 12 (birandom efficient solution at $\alpha_{i}$-levels). Suppose a feasible solution $x^{*}$ of problem (17) satisfies

$$
\mathrm{Ch}^{e}\left\{f_{i}\left(x^{*}, \xi\right) \geq \bar{f}_{i}\left(x^{*}\right)\right\} \geq \alpha_{i}, \quad i=1,2, \ldots, m,
$$

where confidence levels $\alpha_{i} \in[0,1] . x^{*}$ is said to be a birandom efficient solution at $\alpha_{i}$-levels to problem (17) if and only if there exists no other feasible solution $x$ such that

$$
\mathrm{Ch}^{e}\left\{f_{i}(x, \xi) \geq \bar{f}_{i}(x)\right\} \geq \alpha_{i}, \quad i=1,2, \ldots, m,
$$

and $\bar{f}_{i}(x) \geq \bar{f}_{i}\left(x^{*}\right)$ for all $i$ and $\bar{f}_{i_{0}}(x)>\bar{f}_{i_{0}}\left(x^{*}\right)$ for at least one $i_{0} \in\{1,2, \ldots, m\}$.

Specially, we consider the linear form of model (17):

$$
\begin{aligned}
& \max \quad\left\{\bar{f}_{1}, \bar{f}_{2}, \ldots, \bar{f}_{m}\right\} \\
& \text { s. } \quad \text { t. } \operatorname{Ch}^{e}\left\{\widetilde{\widetilde{c}}_{i}^{T} x \geq \bar{f}_{i}\right\} \geq \alpha_{i}, \quad i=1,2, \ldots, m, \\
& \operatorname{Ch}^{e}\left\{\widetilde{\widetilde{a}}_{r}^{T} x \leq \tilde{\widetilde{b}}_{r}\right\} \geq \beta_{r}, \quad r=1,2, \ldots, p, \\
& \quad x \in D,
\end{aligned}
$$

where $\widetilde{\widetilde{c}}_{i}=\left(\widetilde{\widetilde{c}}_{i 1}, \widetilde{\widetilde{c}}_{i 2}, \ldots, \widetilde{\widetilde{c}}_{i n}\right)^{T}$ and $\tilde{\widetilde{a}}_{r}=\left(\tilde{\widetilde{a}}_{r 1}, \widetilde{\widetilde{a}}_{r 2}, \ldots, \widetilde{\widetilde{a}}_{r n}\right)^{T}$ are birandom vectors, and $\tilde{\widetilde{b}}_{r}$ are birandom variables, $i=1,2$, $\ldots, m, r=1,2, \ldots, p$.

It follows from Theorem 10 that model (21) can be rewritten as

$$
\begin{array}{r}
\max \quad\left\{\bar{f}_{1}, \bar{f}_{2}, \ldots, \bar{f}_{m}\right\} \\
\text { s.t. } \quad \operatorname{Pr}\left\{\omega \in \Omega \mid \operatorname{Pr}\left\{\widetilde{\widetilde{c}}_{i}^{T}(\omega) x \geq \bar{f}_{i}\right\} \geq \alpha_{i}\right\} \geq \alpha_{i}, \\
i=1,2, \ldots, m, \\
\operatorname{Pr}\left\{\omega \in \Omega \mid \operatorname{Pr}\left\{\widetilde{\widetilde{a}}_{r}^{T}(\omega) x \geq \widetilde{\widetilde{b}}_{r}\right\} \geq \beta_{r}\right\} \geq \beta_{r}, \\
r=1,2, \ldots, p, \\
x \in D .
\end{array}
$$


Lemma 13. Assume that birandom vector $\widetilde{\widetilde{c}}_{i}(\omega)=\left(\widetilde{\widetilde{c}}_{i 1}(\omega)\right.$, $\left.\widetilde{\tilde{c}}_{i 2}(\omega), \ldots, \widetilde{\tilde{c}}_{i n}(\omega)\right)^{T}$ follows normal distribution with mean vector $\widetilde{c}_{i}(\omega)$ and positive definite covariance matrix $V_{i}^{c}$, denoted by $\widetilde{\widetilde{c}}_{i}(\omega) \sim \mathcal{N}\left(\widetilde{c}_{i}(\omega), V_{i}^{c}\right) . \widetilde{c}_{i}(\omega)$ is a normal random vector with mean vector $\mu_{i}^{c}$ and positive definite covariance matrix $V_{i}^{\prime c}$, written as $\widetilde{c}_{i}(\omega) \sim \mathcal{N}\left(\mu_{i}^{c}, V_{i}^{\prime c}\right)$. If $\widetilde{\tilde{c}}_{i 1}(\omega), \widetilde{\widetilde{c}}_{i 2}(\omega), \ldots, \widetilde{\widetilde{c}}_{i n}(\omega)$ are independent birandom variables, then $\operatorname{Pr}\left\{\omega \mid \operatorname{Pr}\left(\widetilde{\widetilde{c}}_{i}(\omega)^{T} x \geq\right.\right.$ $\left.\left.\bar{f}_{i}\right) \geq \alpha_{i}\right\} \geq \alpha_{i}$ holds if and only if

$$
\mu_{i}^{c T} x+\Phi^{-1}\left(1-\alpha_{i}\right) \sqrt{x^{T} V_{i}^{c} x}+\Phi^{-1}\left(1-\alpha_{i}\right) \sqrt{x^{T} V_{i}^{c^{\prime}} x} \geq \bar{f}_{i},
$$

where $\Phi$ is the standardized normal distribution.

Proof. Let $y_{i}=\tilde{\tilde{c}}_{i}^{T}(\omega) x-\bar{f}_{i}$; then $y_{i}$ follows normal distribution with the following expected value and variance:

$$
E\left[y_{i}\right]=\widetilde{c}_{i}(\omega)^{T} x-\bar{f}_{i}, \quad \operatorname{Var}\left[y_{i}\right]=x^{T} V_{i}^{c} x,
$$

where $\widetilde{c}_{i}(\omega)=\left(\widetilde{c}_{i 1}(\omega), \widetilde{c}_{i 2}(\omega), \ldots, \widetilde{c}_{i n}(\omega)\right)^{T}$. We note that

$$
\frac{\left(\tilde{\tilde{c}}_{i}(\omega)^{T} x-\bar{f}_{i}\right)-\left(\widetilde{c}_{i}(\omega)^{T} x-\bar{f}_{i}\right)}{\sqrt{x^{T} V_{i}^{c} x}}
$$

must be standardized normally distributed. The inequality $\tilde{\widetilde{c}}_{i}(\omega)^{T} x \geq \bar{f}_{i}$ is equivalent to

$$
\frac{\left(\widetilde{\tilde{c}}_{i}(\omega)^{T} x-\bar{f}_{i}\right)-\left(\widetilde{c}_{i}(\omega)^{T} x-\bar{f}_{i}\right)}{\sqrt{x^{T} V_{i}^{c} x}} \geq-\frac{c_{i}(\omega)^{T} x-\bar{f}_{i}}{\sqrt{x^{T} V_{i}^{c} x}} .
$$

Let $\eta=\left(\left(\widetilde{\widetilde{c}}_{i}(\omega)^{T} x-\bar{f}_{i}\right)-\left(\widetilde{c}_{i}(\omega)^{T} x-\bar{f}_{i}\right)\right) / \sqrt{x^{T} V_{i}^{c} x}$, which is standardized normally distributed. Then we have

$$
\begin{aligned}
& \operatorname{Pr}\left\{\widetilde{\tilde{c}}_{i j}(\omega)^{T} x \geq \bar{f}_{i}\right\} \geq \alpha_{i} \\
& \Longleftrightarrow \operatorname{Pr}\left\{\eta \geq \frac{\bar{f}_{i}-\widetilde{c}_{i}(\omega)^{T} x}{\sqrt{x^{T} V_{i}^{c} x}}\right\} \geq \alpha_{i} \\
& \Longleftrightarrow 1-\operatorname{Pr}\left\{\eta \leq \frac{\bar{f}_{i}-\widetilde{c}_{i}(\omega)^{T} x}{\sqrt{x^{T} V_{i}^{c} x}}\right\} \geq \alpha_{i} \\
& \Longleftrightarrow \operatorname{Pr}\left\{\eta \leq \frac{\bar{f}_{i}-\widetilde{c}_{i}(\omega)^{T} x}{\sqrt{x^{T} V_{i}^{c} x}}\right\} \leq 1-\alpha_{i} \\
& \Longleftrightarrow \Phi\left(\frac{\bar{f}_{i}-\widetilde{c}_{i}(\omega)^{T} x}{\sqrt{x^{T} V_{i}^{c} x}}\right) \leq 1-\alpha_{i} \\
& \Longleftrightarrow \frac{\bar{f}_{i}-\widetilde{c}_{i}(\omega)^{T} x}{\sqrt{x^{T} V_{i} x} \leq \Phi^{-1}\left(1-\alpha_{i}\right)} \\
& \Longleftrightarrow \widetilde{c}_{i}(\omega)^{T} x+\Phi^{-1}\left(1-\alpha_{i}\right) \sqrt{x^{T} V_{i}^{c} x} \geq \bar{f}_{i} .
\end{aligned}
$$

Thus

$$
\begin{gathered}
\operatorname{Pr}\left\{\omega \mid \operatorname{Pr}\left\{\tilde{\tilde{c}}_{i j}(\omega)^{T} x \geq \bar{f}_{i}\right\} \geq \alpha_{i}\right\} \geq \alpha_{i} \\
\Longleftrightarrow \operatorname{Pr}\left\{\widetilde{c}_{i}(\omega)^{T} x+\Phi^{-1}\left(1-\alpha_{i}\right) \sqrt{x^{T} V_{i}^{c} x} \geq \bar{f}_{i}\right\} \geq \alpha_{i} \\
\Longleftrightarrow \operatorname{Pr}\left\{\left(\left(\widetilde{c}_{i}(\omega)^{T} x+\Phi^{-1}\left(1-\alpha_{i}\right) \sqrt{x^{T} V_{i}^{c} x}-\bar{f}_{i}\right)\right.\right. \\
\left.-\left(\mu_{i}^{c T} x+\Phi^{-1}\left(1-\alpha_{i}\right) \sqrt{x^{T} V_{i}^{c} x}-\bar{f}_{i}\right)\right) \\
\times\left(\sqrt{x^{c T} V_{i}^{c^{\prime}} x}\right)^{-1} \\
\left.\geq-\frac{\mu_{i}^{c T} x+\Phi^{-1}\left(1-\alpha_{i}\right) \sqrt{x^{T} V_{i}^{c} x}-\bar{f}_{i}}{\sqrt{x^{T} V_{i}^{c^{\prime}} x}}\right\} \geq \alpha_{i} \\
\Longleftrightarrow \mu_{i}^{c T} x+\Phi^{-1}\left(1-\alpha_{i}\right) \sqrt{x^{T} V_{i}^{c} x}+\Phi^{-1}\left(1-\alpha_{i}\right) \\
\times \sqrt{x^{T} V_{i}^{c^{\prime}} x} \geq \bar{f}_{i} .
\end{gathered}
$$

This completes the proof.

Lemma 14 (see [21]). Assume that birandom vector $\tilde{\tilde{a}}_{r}(\omega)=$ $\left(\tilde{\tilde{a}}_{r 1}(\omega), \tilde{\tilde{a}}_{r 2}(\omega), \ldots, \widetilde{\tilde{a}}_{i n}(\omega)\right)^{T}$ follows normal distribution with mean vector $\widetilde{a}_{r}(\omega)$ and positive definite covariance matrix $V_{r}^{a}$, written as $\widetilde{a}_{r}(\omega) \sim \mathcal{N}\left(\widetilde{a}_{r}(\omega), V_{r}^{a}\right) . \widetilde{a}_{r}(\omega)$ is a normally distributed random variable, written as $\tilde{a}_{r}(\omega) \sim$ $\mathcal{N}\left(\mu_{r}^{a}, V_{r}^{\prime a}\right)$. Birandom variable $\widetilde{\widetilde{b}}_{r}(\omega)$ follows normal distribution with mean value $\widetilde{b}_{r}(\omega)$ and variance $\left(\sigma_{r}^{b}\right)^{2}$, denoted by $\widetilde{\widetilde{b}}_{r}(\omega) \sim \mathcal{N}\left(\widetilde{b}_{r}(\omega),\left(\sigma_{r}^{b}\right)^{2}\right) . \widetilde{b}_{r}(\omega)$ is a normally distributed random variable, written as $\widetilde{b}_{r}(\omega) \sim \mathcal{N}\left(\mu_{r}^{b},\left(\sigma_{r}^{\prime b}\right)^{2}\right)$. If $\tilde{\tilde{a}}_{r 1}(\omega), \tilde{\tilde{a}}_{r 2}(\omega), \ldots, \widetilde{\tilde{a}}_{r n}(\omega), \widetilde{\tilde{b}}_{r}(\omega)$ are independent birandom variables, then $\operatorname{Ch}^{e}\left\{\tilde{\tilde{a}}_{r}^{T} x \leq \tilde{\widetilde{b}}_{r}\right\} \geq \beta_{r}$ holds if and only if

$$
\begin{aligned}
& u_{r}^{a T} x+\Phi^{-1}\left(\beta_{r}\right) \sqrt{x^{T} V_{r}^{a} x+\left(\sigma_{r}^{b}\right)^{2}} \\
& \quad+\Phi^{-1}\left(\beta_{r}\right) \sqrt{x^{T} V_{r}^{a^{\prime}} x+\left(\sigma_{r}^{b^{\prime}}\right)^{2}} \leq \mu_{r}^{b}, \quad r=1,2, \ldots, p,
\end{aligned}
$$

where $\beta_{r}$ are predetermined confidence levels, $r=1,2, \ldots, p$.

It follows from Lemmas 13 and 14 that model (21) is equivalent to the following crisp multiobjective programming model: 


$$
\begin{aligned}
\max \quad\left\{\bar{f}_{1}, \bar{f}_{2}, \ldots, \bar{f}_{m}\right\} & \\
\text { s.t. } \quad \bar{f}_{i} \leq \mu_{i}^{c T} x+\Phi^{-1}\left(1-\alpha_{i}\right) \sqrt{x^{T} V_{i}^{c} x} & \\
+\Phi^{-1}\left(1-\alpha_{i}\right) \sqrt{x^{T} V_{i}^{c^{\prime}} x}, & i=1,2, \ldots, m, \\
x & \in X,
\end{aligned}
$$

or equivalently

$$
\max _{x \in X}\left\{F_{1}(x), F_{2}(x), \ldots, F_{m}(x)\right\},
$$

where $F_{i}(x):=\mu_{i}^{c T} x+\Phi^{-1}\left(1-\alpha_{i}\right) \sqrt{x^{T} V_{i}^{c} x}+\Phi^{-1}(1-$ $\left.\alpha_{i}\right) \sqrt{x^{T} V_{i}^{c^{\prime}} x}, i=1,2, \ldots, m$, and $X:=\left\{x \mid u_{r}^{a T} x+\right.$ $\Phi^{-1}\left(\beta_{r}\right) \sqrt{x^{T} V_{r}^{a} x+\left(\sigma_{r}^{b}\right)^{2}}+\Phi^{-1}\left(\beta_{r}\right) \sqrt{x^{T} V_{r}^{a^{\prime}} x+\left(\sigma_{r}^{b^{\prime}}\right)^{2}} \leq \mu_{r}^{b}$, $x \in D, r=1,2, \ldots, p\}$.

\section{Solution Method}

If model (17) satisfies the conditions for Lemmas 13 and 14, then model (17) can be converted into a crisp multiobjective programming model. Many classical methods, such as the goal programming method, the interactive method, and the weighted sum method, can be used to solve it. However, for a general case, it is a difficult to convert the general model into its deterministic equivalent for the predetermined confidence levels. In order to handle the birandom objective functions and to check the birandom equilibrium chance constraints, we used a birandom simulation technique as this method is similar to a stochastic simulation but more complicated and time consuming.

For the following constraints,

$$
\operatorname{Pr}\left\{\omega \in \Omega \mid \operatorname{Pr}\left\{\tilde{\widetilde{c}}_{i}(\omega)^{T} x \geq \bar{f}_{i}\right\} \geq \alpha_{i}\right\} \geq \alpha_{i}, \quad i=1,2, \ldots, m ;
$$

in view of the purpose of maximizing $\bar{f}_{i}$, we should find the maximal $\bar{f}_{i}$ such that (32) holds for a given $x$. It suffices to estimate the maximal value of $\bar{f}_{i}$ such that the probability of the following random event

$$
\left\{\omega \in \Omega \mid \operatorname{Pr}\left\{\tilde{\tilde{c}}_{i}(\omega)^{T} x \geq \bar{f}_{i}\right\} \geq \alpha_{i}\right\}
$$

is not less than $\alpha_{i}$.

First, we generate $N$ independent vectors $\omega^{k}=\left(\omega_{1}^{k}\right.$, $\left.\omega_{2}^{k}, \ldots, \omega_{n}^{k}\right)$ from $\Omega$ according to distribution function. Then $\widetilde{\widetilde{c}}_{i}\left(\omega^{k}\right)$ are random vectors, $k=1,2, \ldots, N$. So we can apply the stochastic simulation to handle

$$
\operatorname{Pr}\left\{\widetilde{\tilde{c}}_{i}\left(\omega^{k}\right)^{T} x\right\} \geq \bar{f}_{i}, \quad i=1,2, \ldots, m .
$$

We define

$$
h\left(x, \widetilde{\widetilde{c}}_{i}\left(\omega^{k}\right)\right)= \begin{cases}1, & \text { if } \widetilde{\tilde{c}}_{i}\left(\omega^{k}\right)^{T} x \geq \bar{f}_{i}, \\ 0, & \text { otherwise }\end{cases}
$$

for $k=1,2, \ldots, N$, which are random variables, and the expected value $E\left[h\left(x, \widetilde{\widetilde{c}}_{i}\left(\omega^{k}\right)\right)\right]=\alpha_{i}$ for all $k$. By the strong law of large numbers, we obtain

$$
\frac{\sum_{k=1}^{N} h\left(x, \tilde{\widetilde{c}}_{i}\left(\omega^{k}\right)\right)}{N} \longrightarrow \alpha_{i}
$$

as $N \rightarrow \infty$. Note that $\sum_{k=1}^{N} h\left(x, \tilde{\widetilde{c}}_{i}\left(\omega^{k}\right)\right)$ is just the number of $\tilde{\tilde{c}}_{i}\left(\omega^{k}\right)$ satisfying $\widetilde{\tilde{c}}_{i}\left(\omega^{k}\right)^{T} x \geq \bar{f}_{i}$ for $i=1,2, \ldots, N$. Thus $\bar{f}_{i}$ is just the $N^{\prime}$ th largest element in the sequence $\left\{\tilde{\bar{c}}_{i}\left(\omega^{1}\right)^{T} x, \widetilde{\tilde{c}}_{i}\left(\omega^{2}\right)^{T} x, \ldots, \tilde{\widetilde{c}}_{i}\left(\omega^{N}\right)^{T} x\right\}$, where $N^{\prime}$ is the integer part of $\alpha_{i} N$. The process to estimate the maximal $\bar{f}_{i}$ such that (32) holds is summarized as follows.

Step 1. Sample $\omega^{1}, \omega^{2}, \ldots, \omega^{N}$ from $\Omega$ according to distribution function.

Step 2. Find the maximal values $\bar{f}_{\text {in }}$ such that $\operatorname{Pr}\left\{\widetilde{\tilde{c}}_{i}^{T}\left(\omega^{k}\right) x \geq\right.$ $\left.\bar{f}_{\text {in }}\right\} \geq \alpha_{i}$ for $n=1,2, \ldots, N$, respectively, by stochastic simulation.

Step 3. Set $N^{\prime}$ as the integer part of $\alpha_{i} N$.

Step 4. Return the $N^{\prime}$ th largest element in $\left\{\bar{f}_{i 1}, \bar{f}_{i 2}, \ldots, \bar{f}_{i N}\right\}$ as $\bar{f}_{i}, i=1,2, \ldots, m$.

For a fixed $x$, we check whether the constraint

$$
\operatorname{Pr}\left\{\omega \mid \operatorname{Pr}\left\{\tilde{\widetilde{a}}_{r}(\omega)^{T} x \leq \tilde{\widetilde{b}}_{r}(\omega)\right\} \geq \beta_{r}\right\} \geq \beta_{r}, \quad r=1,2, \ldots, p,
$$

holds by following process.

Step 1. Set $N^{\prime}=0$.

Step 2. Generate $\omega=\left(\omega_{1}, \omega_{2}, \ldots, \omega_{n}\right)^{T}$ from $\Omega$ according to distribution function.

Step 3. Calculate $\operatorname{Pr}\left\{\tilde{\tilde{a}}_{r}(\omega)^{T} x \leq \widetilde{\widetilde{b}}_{r}(\omega)\right\}$ by stochastic simulation.

Step 4. If $\operatorname{Pr}\left\{\tilde{\tilde{a}}_{r}(\omega)^{T} x \leq \tilde{\widetilde{b}}_{r}(\omega)\right\} \geq \beta_{r}$, then $N^{\prime}=N^{\prime}+1$.

Step 5. Repeat Step 2 to Step $4 N$ times.

Step 6. If $N_{r}^{\prime} / N \geq \beta_{r}$, return $x$ is feasible, or else $x$ is infeasible.

Genetic algorithms (GAs) are stochastic search methods for optimization problems based on the mechanics of natural selection and natural genetics. They have been applied to different sectors for both technical and management problems and have shown good performance [22-26]. In this paper, the birandom simulation technique is embedded into a GA to develop the birandom simulation-based GA. The overall procedure of the birandom simulation-based GA for solving the birandom programming models is shown in Figure 2. The main parts of the algorithm are stated in more detail as follows. 


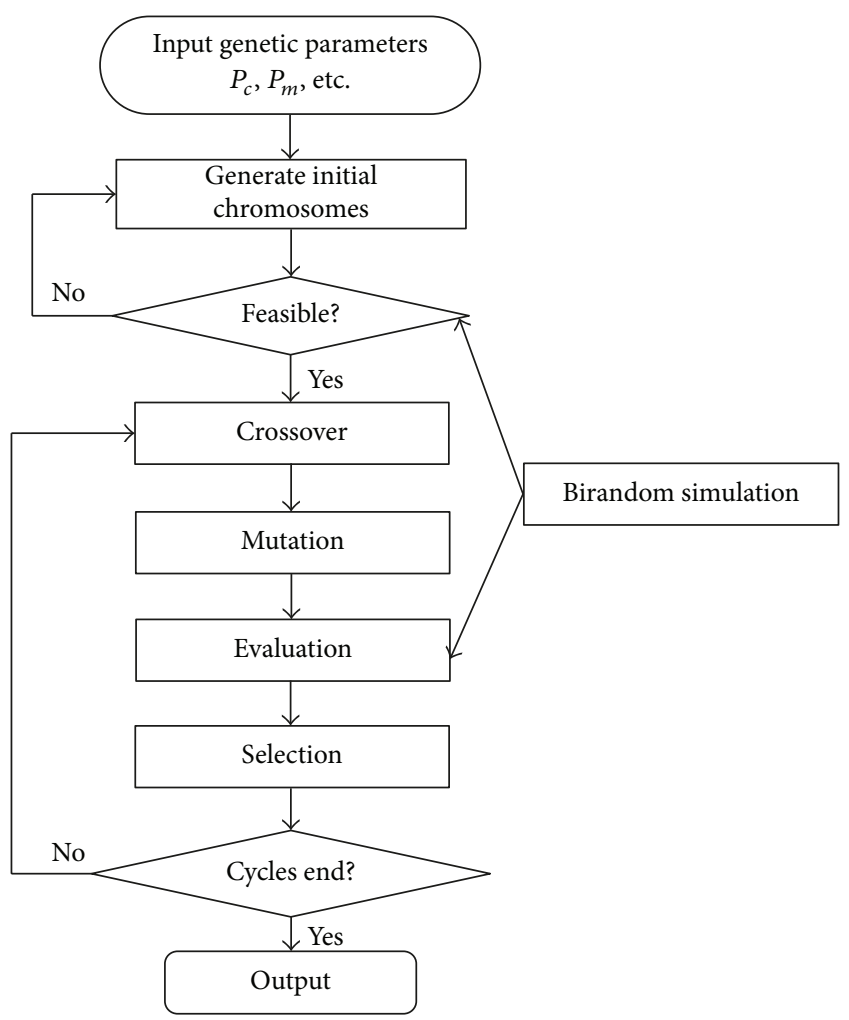

Figure 2: The flowchart of the birandom simulation-based GA.

(1) Representation: a vector $x=\left(x_{1}, x_{2}, \ldots, x_{n}\right)$ is chosen as a chromosome to represent a solution to the optimization problem.

(2) Handling the constraints: to ensure the chromosomes generated by genetic operators are feasible, we can use the technique of birandom simulation to check them.

(3) Initializing process: suppose that the decision maker is able to predetermine a region which contains the feasible set. Randomly generate a vector $x$ from this region until a feasible one is accepted as a chromosome. Repeat the above process $N_{\text {pop-size }}$ times; then we have $N_{\text {pop-size }}$ initial feasible chromosomes $x^{1}, x^{2}, \ldots, x^{N_{\text {pop-size }}}$. In general, the larger the number of population size $N_{\text {pop-size }}$ is, the better the result is. However, the computation time will increase with $N_{\text {pop-size }}$ increasing. In the paper, we set $N_{\text {pop-size }}$ from 20 to 40 .

(4) Evaluation function: let eval $(x)$ equal weighted sum of all objectives; that is,

$$
\operatorname{eval}(x)=\sum_{i=1}^{m} w_{i} f_{i}
$$

where $w_{i}$ are random numbers in $[0,1]$, representing the weight for objective $f_{i}, i=1,2, \ldots, m$. The random weightsum approach explores the entire solution space in order to avoid local optima and thus gives a uniform chance to search all the possible solutions. All chromosomes are arranged from large to small according to their objective function values. In other words, after rearrangement, $x^{1}$ is the best chromosome, and $x^{N_{\text {pop-size }}}$ is the worst one.
(5) Selection process: the selection process is based on spinning the roulette wheel $N_{\text {pop-size }}$ times. Each time a single chromosome for a new population is selected in the following way: calculate the cumulative probability $q_{i}$ for each chromosome $x^{i}$ :

$$
q_{0}=0, \quad q_{i}=\sum_{j=1}^{i} \operatorname{eval}\left(x^{j}\right), \quad i=1,2, \ldots, N_{\text {pop-size }}
$$

Generate a random number $r$ in $\left[0, q_{N_{\text {pop-size }}}\right]$ and select the $i$ th chromosome $x_{i}$ such that $q_{i-1}<r \leq q_{i}, 1 \leq i \leq$ $N_{\text {pop-size }}$. Repeat the above process $N_{\text {pop-size }}$ times and we obtain $N_{\text {pop-size }}$ copies of chromosomes.

(6) Crossover operation: generate a random number $c$ from the open interval $(0,1)$ and the chromosome $x^{i}$ is selected as a parent provided that $c<P_{c}$, where parameter $P_{c}$ is the probability of crossover operation. Repeat this process $N_{\text {pop-size }}$ times and $P_{c} \cdot N_{\text {pop-size }}$ chromosomes are expected to be selected to undergo the crossover operation. The crossover operator on $x^{1}$ and $x^{2}$ will produce two children $y^{1}$ and $y^{2}$ as follows:

$$
y^{1}=c x^{1}+(1-c) x^{2}, \quad y^{2}=c x^{2}+(1-c) x^{2}
$$

If both children are feasible, then we replace the parents with them, or else we keep the feasible one if it exists. Repeat the above operation until two feasible children are obtained or a given number of cycles is finished.

Let us consider the setting of probability of crossover $P_{c}$. It is obvious that larger crossover probability results in reaching larger solution space, and then it helps reduce the chance of stopping at nonoptimal solution. However, too large $P_{c}$ will result in considerable time consuming because of too much searching in unnecessary solution space. In the paper, we set $P_{c}$ from 0.2 to 0.4 .

(7) Mutation operation: similar to the crossover process, the chromosome $x^{i}$ is selected as a parent to undergo the mutation operation provided that random number $m<P_{m}$, where parameter $P_{m}$ is the probability of mutation operation. $P_{\lambda_{i}} \cdot N_{\text {pop-size }}$ are expected to be selected after repeating the process $N_{\text {pop-size }}$ times. Suppose that $x^{1}$ is chosen as a parent. Choose a mutation direction $\mathbf{d} \in \mathbf{R}^{\mathbf{n}}$ randomly. Replace $x$ with $x+M \cdot \mathbf{d}$ if $x+M \cdot \mathbf{d}$ is feasible; otherwise we set $M$ as a random number between 0 and $M$ until it is feasible or a given number of cycles are finished. Here, $M$ is a sufficiently large positive number.

$P_{m}$ controls the proportion of new genes generating in population. If $P_{m}$ is too small, it will be difficult for some effective genes to be selected. On the contrary, if $P_{m}$ is too large, that is, there exists too much random change, then offsprings may lose good characteristics inherited from their parents. Thus, the algorithm will lose the learning ability from the past searching. In the paper, we set $P_{m}$ from 0.2 to 0.4 .

After running a given number of cycles of above birandom simulation-based genetic algorithm, the best chromosome can be regarded as the optimal solution. 


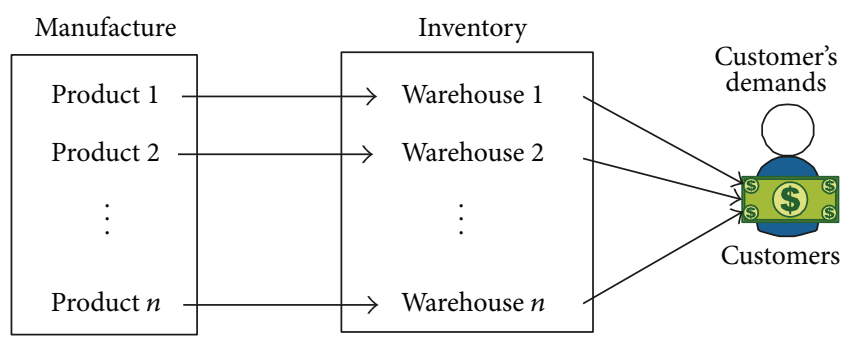

FIgURE 3: The inventory system of H Chain Co., Ltd.

\section{Practical Application for H Chain Co., Ltd.}

A real-world inventory problem is discussed in this section to illustrate the effectiveness of the theoretical results in this paper.

4.1. Key Problem Statement and Model Formulation. The problem is from H Chain Co., Ltd., which is one of largest chain enterprises in southwest of China. It operates a variety of products, such as eggs, grains, milk, and meat. Inventory management is an important part of company management. The inventory system of $\mathrm{H}$ Chain Co., Ltd. is illustrated by Figure 3.

A new executive is assigned to the management of four product items: eggs, dairy products, grains, and meat products. The executive needs to make an effective inventory strategy to reduce costs and increase profit.

4.1.1. Assumptions. In order to develop the mathematical model for the inventory problem, there are some assumptions made, which are as follows. (1) This is an inventory model with a finite time horizon. (2) There are multiple items in this inventory system. (3) Average demand rates are continuous and constant. (4) Deterioration rates are constant. (5) Shortage is allowed. (6) Initial inventory quantities are not zeros; (7) The inventory system uses a continuous review fixedorder-quantity strategy. (8) The lead time is proportional to the order quantity. (9) Fixed-ordering costs are constant. (10) Unit price, unit acquisition cost, holding cost per unit per unit time and shortage cost per unit per unit time are birandom variables. (11) Available total storage space is limited. (12) Available total budget is limited. (13) The inventory system uses nonintegrated management.

4.1.2. Notations. The following notations are used to describe this inventory model:

$n$ is the number of items;

$W_{i}$ is the available storage space of warehouse of product $i, i=1,2, \ldots, n$;

$B$ is the available total budgetary cost;

$K_{i}$ is the required storage area per unit quantity $i, i=$ $1,2, \ldots, n$;

$A_{i}$ is the fixed-ordering cost of product $i, i=$ $1,2, \ldots, n$;

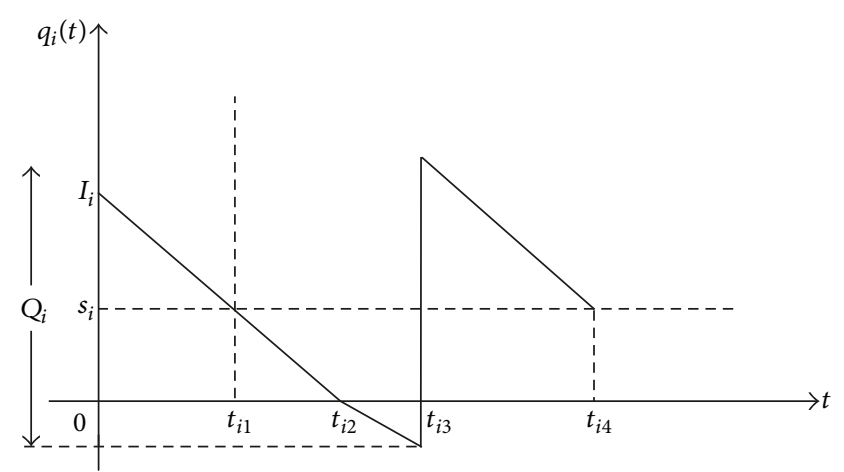

FIGURE 4: Inventory level of product $i$ at time $t$.

$D_{i}$ is the average demand rate of product $i, i=$ $1,2, \ldots, n$;

$I_{i}$ is the initial demand rate of product $i, i=1,2, \ldots, n$;

$a_{i}$ is the average deteriorating rate of product $i, i=$ $1,2, \ldots, n$;

$l_{i}$ is the lead time for unit product $i, i=1,2, \ldots, n$;

$\widetilde{\widetilde{p}}_{i}$ is the unit selling price of product $i$ (birandom variable), $i=1,2, \ldots, n$;

$\widetilde{\widetilde{c}}_{i 1}$ is the unit acquisition cost of product $i$ (birandom variable), $i=1,2, \ldots, n$;

$\widetilde{\widetilde{c}}_{i 2}$ is the unit shortage cost of product $i$ (birandom variable), $i=1,2, \ldots, n$;

$s_{i}$ is the reorder point of product $i$ (decision variable), $i=1,2, \ldots, n$;

$Q_{i}$ is the order quantity of product $i$ (decision variable), $i=1,2, \ldots, n$;

$\widetilde{\widetilde{N}}_{i}$ is the net revenue of product $i, i=1,2, \ldots, n$;

$\widetilde{\widetilde{C}}_{i 1}$ is the accquisition cost of product $i, i=1,2, \ldots, n$;

$\widetilde{\widetilde{C}}_{i 2}$ is the holding cost of product $i, i=1,2, \ldots, n$;

$\widetilde{\widetilde{C}}_{i 3}$ is the shortage cost of product $i, i=1,2, \ldots, n$;

$\widetilde{\widetilde{T P}}_{i}$ is the average profit of product $i, i=1,2, \ldots, n$.

4.2. Model Formulation. It follows from the assumptions above that the inventory level $q_{i}(t)$ of product $i$ at time $t$ can be illustrated by Figure 4 .

In Figure $4, t_{i 1}$ is the time of order point for product $i$; $t_{i 2}$ is the time when inventory level of product $i$ reduces to zero; $t_{i 3}$ is the time when replenishment of product $i$ arrives; $t_{i 4}$ is the time when inventory level of product $i$ reduces to reorder point. 
From Figure 4, we have

$$
\begin{aligned}
t_{i 1}= & \frac{I_{i}-s_{i}}{D_{i}+a_{i}}, \\
t_{i 2}= & \frac{I_{i}}{D_{i}+a_{i}}, \\
t_{i 3}= & t_{i 1}+l_{i} Q_{i}=\frac{I_{i}-s_{i}}{D_{i}+a_{i}}+l_{i} Q_{i}, \\
t_{i 4}= & t_{i 3}+\frac{Q_{i}-s_{i}-\left(t_{i 3}-t_{i 2}\right) D_{i}}{D_{i}+a_{i}} \\
= & \frac{I_{i}-s_{i}}{D_{i}+a_{i}}+l_{i} Q_{i}+\frac{Q_{i}-s_{i}-\left(-s_{i} /\left(D_{i}+a_{i}\right)+l_{i} Q_{i}\right) D_{i}}{D_{i}+a_{i}} \\
= & \frac{I_{i}+Q_{i}-2 s_{i}-\left(-s_{i} /\left(D_{i}+a_{i}\right)+l_{i} Q_{i}\right) D_{i}}{D_{i}+a_{i}}+l_{i} Q_{i} .
\end{aligned}
$$

For product $i$, the total profit from initial time 0 to $t_{i 4}$ equals the net revenue minus total cost, including fixedordering cost, acquisition cost, holding cost, and shortage cost. The net revenue is equal to unit price multiplied by sale quantity, that is,

$$
\widetilde{\widetilde{N}}_{i}=\widetilde{\widetilde{p}}_{i}\left(t_{i 2}+t_{i 4}-t_{i 3}\right) D_{i}=\widetilde{\widetilde{p}}_{i}\left(\frac{I_{i}+Q_{i}-s_{i}-l_{i} Q_{i} D_{i}}{D_{i}+a_{i}}\right) D_{i} .
$$

The acquisition cost is equal to unit acquisition cost multiplied ordering quantity; that is,

$$
\widetilde{\widetilde{C}}_{i 1}=\widetilde{\widetilde{c}}_{i 1} Q_{i}
$$

The total holding cost consists of holding cost from 0 to $t_{i 2}$ and holding cost from $t_{i 3}$ to $t_{i 4}$; that is,

$$
\begin{aligned}
\widetilde{\widetilde{C}}_{i 2} & =\widetilde{\widetilde{c}}_{i 2}\left(\int_{0}^{t_{i 2}} q_{i}(t) d t+\int_{t_{i 3}}^{t_{i 4}} q_{i}(t) d t\right) \\
& =\widetilde{\widetilde{c}}_{i 2}\left(\frac{I_{i}}{2} t_{i 2}+\frac{Q_{i}-\left(t_{i 3}-t_{i 2}\right) D_{i}+s_{i}}{2}\left(t_{i 4}-t_{i 3}\right)\right) \\
& =\widetilde{\widetilde{c}}_{i 2}\left(\frac{I_{i}^{2}+\left(Q_{i}-l_{i} Q_{i} D_{i}\right)^{2}-s_{i}^{2}}{2\left(D_{i}+a_{i}\right)}\right) .
\end{aligned}
$$

The shortage cost occurs from $t_{i 2}$ to $t_{i 3}$; that is,

$$
\widetilde{\widetilde{C}}_{i 3}=\widetilde{\widetilde{c}}_{i 3} \int_{t_{i 3}}^{t_{i 3}} D_{i} d t=\widetilde{\widetilde{c}}_{i 3} \frac{\left(t_{i 3}-t_{i 2}\right) D_{i}}{2}=\frac{\widetilde{\widetilde{c}}_{i 3} l_{i} Q_{i} D_{i}}{2} .
$$

The total profit of product $i$ from $t_{i 2}$ to $t_{i 4}$ is $\widetilde{\widetilde{N}}_{i 0}-A_{i}-$ $\widetilde{\widetilde{C}}_{i 1}-\widetilde{\widetilde{C}}_{i 2}-\widetilde{\widetilde{C}}_{i 3}$. Then the average total profit of product $i$ is formulated as

$$
\widetilde{\widetilde{T P}}_{i}=\frac{\widetilde{\widetilde{N}}_{i}-A_{i}-\widetilde{\widetilde{C}}_{i 1}-\widetilde{\widetilde{C}}_{i 2}-\widetilde{\widetilde{C}}_{i 3}}{t_{i 4}} .
$$

It is noted that $\widetilde{\widetilde{T P}}_{i}$ can not be maximized due to birandomness. By utilizing the idea of Charnes and Cooper [17], $\bar{f}_{i}$ is maximized which satisfies

$$
\mathrm{Ch}^{e}\left\{\widetilde{\widetilde{T P}}_{i} \geq \bar{f}_{i}\right\} \geq \alpha_{i}
$$

where $\operatorname{Ch}^{e}\{\cdot\}$ is the equilibrium chance and $\alpha_{i}$ is given confidence level.

The space of product $i$ ordered should not exceed the available storage space of warehouse; that is,

$$
K_{i} Q_{i} \leq W_{i}
$$

All the costs should not exceed the available total budgetary cost; that is, $\sum_{i=1}^{m}\left(\widetilde{\widetilde{C}}_{i 1}+\widetilde{\widetilde{C}}_{i 2}+\widetilde{\widetilde{C}}_{i 3}+\widetilde{\widetilde{C}}_{i 4}\right) \leq B$. It is noted that the constraint is meaningless mathematically due to the existence of birandom variable. We use equilibrium chanceconstrained technique to make it meaningful mathematically, that is,

$$
\mathrm{Ch}^{e}\left\{\sum_{i=1}^{n}\left(A_{i}+\widetilde{\widetilde{C}}_{i 1}+\widetilde{\widetilde{C}}_{i 2}+\widetilde{\widetilde{C}}_{i 3}\right) \leq B\right\} \leq \beta,
$$

where $\beta$ is the confidence level predetermined by the decision maker.

Since shortage is allowed, the order quantity of product $i$ should not be less than reorder point $s_{i}$; that is,

$$
Q_{i} \geq s_{i}
$$

In addition, It is natural to require the nonnegativity of decision variable; that is,

$$
Q_{i} \geq 0, \quad s_{i} \geq 0 .
$$

By integration of (47)-(51), we can formulate the programming model of the inventory problem as

$$
\begin{array}{ll}
\max & \quad\left\{\bar{f}_{1}, \bar{f}_{2}, \ldots, \bar{f}_{m}\right\} \\
\text { s.t. } \quad & \mathrm{Ch}^{e}\left\{\widetilde{\widetilde{T C}}_{i} \geq \bar{f}_{i}\right\} \geq \alpha_{i}, \quad i=1,2, \ldots, n, \\
& K_{i} Q_{i} \leq W_{i}, \quad i=1,2, \ldots, n, \\
& Q_{i} \geq s_{i}, \quad i=1,2, \ldots, n, \\
& \mathrm{Ch}^{e}\left\{\sum_{i=1}^{n}\left(A_{i}+\widetilde{\widetilde{C}}_{i 1}+\widetilde{\widetilde{C}}_{i 2}+\widetilde{\widetilde{C}}_{i 3}\right) \leq B\right\} \leq \beta, \\
Q_{i} \geq 0, \quad s_{i} \geq 0, \quad i=1,2, \ldots, n .
\end{array}
$$

4.3. Data and Compaction Result. All the data are from the marketing section of $\mathrm{H}$ Chain Co., Ltd. These data can be divided into two different groups: determined parameters and birandom parameters. Determined parameters include $W_{i}, B, K_{i}, A_{i}, D_{i}, I_{i}, a_{i}$, and $l_{i}$. The available total budgetary cost $B=250000$ yuan. Except for $B$, all determined parameters are listed in Table 1 . Birandom variables including 
TABLE 1: Determined parameters.

\begin{tabular}{|c|c|c|c|c|c|c|c|}
\hline & $W_{i}\left(\mathrm{~m}^{3}\right)$ & $K_{i}\left(\mathrm{~m}^{3} / \mathrm{kg}\right)$ & $A_{i}$ (yuan) & $D_{i}$ (kg/day) & $I_{i}(\mathrm{~kg})$ & $a_{i}$ (kg/day) & $l_{i}($ day $/ \mathrm{kg})$ \\
\hline Eggs & 5.00 & $0.50 \times 10^{-3}$ & 500 & 500 & 1200 & 10 & $1 \times 10^{-3}$ \\
\hline Dairy products & 5.00 & $0.80 \times 10^{-3}$ & 1000 & 500 & 2000 & 20 & $2 \times 10^{-3}$ \\
\hline Grains & 20.00 & $0.40 \times 10^{-3}$ & 2000 & 1000 & 3000 & 10 & $2 \times 10^{-3}$ \\
\hline Meat products & 10.00 & $0.50 \times 10^{-3}$ & 1000 & 1000 & 2000 & 50 & $4 \times 10^{-3}$ \\
\hline
\end{tabular}

TABLE 2: Birandom variables.

\begin{tabular}{|c|c|c|c|c|}
\hline & $\widetilde{\tilde{p}}_{i}$ (yuan $/ \mathrm{kg}$ ) & $\widetilde{\tilde{c}}_{i 1}($ yuan $/ \mathrm{kg})$ & $\widetilde{\widetilde{c}}_{i 2}($ yuan $/ \mathrm{kg})$ & $\widetilde{\widetilde{c}}_{i 3}($ yuan $/ \mathrm{kg})$ \\
\hline Eggs & $\mathcal{N}(\tilde{\mu}, 1), \tilde{\mu} \sim \mathcal{N}(16,1)$ & $\mathcal{N}(\widetilde{\mu}, 1), \widetilde{\mu} \sim \mathcal{N}(12,1)$ & $\mathcal{N}(\widetilde{\mu}, 0.01), \widetilde{\mu} \sim \mathcal{N}(0.10,0.01)$ & $\mathcal{N}(\widetilde{\mu}, 1), \widetilde{\mu} \sim \mathcal{N}(15,1)$ \\
\hline Dairy products & $\mathcal{N}(\tilde{\mu}, 1), \tilde{\mu} \sim \mathcal{N}(20,1)$ & $\mathcal{N}(\tilde{\mu}, 1), \tilde{\mu} \sim \mathcal{N}(16,1)$ & $\mathcal{N}(\tilde{\mu}, 0.01), \tilde{\mu} \sim \mathcal{N}(0.10,0.01)$ & $\mathscr{N}(\widetilde{\mu}, 1), \tilde{\mu} \sim \mathscr{N}(18,1)$ \\
\hline Grains & $\mathcal{N}(\tilde{\mu}, 1), \tilde{\mu} \sim \mathcal{N}(8,1)$ & $\mathcal{N}(\tilde{\mu}, 1), \tilde{\mu} \sim \mathcal{N}(4,1)$ & $\mathcal{N}(\tilde{\mu}, 0.01), \tilde{\mu} \sim \mathcal{N}(0.10,0.01)$ & $\mathcal{N}(\tilde{\mu}, 1), \tilde{\mu} \sim \mathcal{N}(6,1)$ \\
\hline Meat products & $\mathcal{N}(\tilde{\mu}, 1), \tilde{\mu} \sim \mathcal{N}(40,1)$ & $\mathcal{N}(\tilde{\mu}, 1), \tilde{\mu} \sim \mathcal{N}(30,1)$ & $\mathcal{N}(\widetilde{\mu}, 0.01), \tilde{\mu} \sim \mathcal{N}(0.10,0.01)$ & $\mathscr{N}(\widetilde{\mu}, 1), \widetilde{\mu} \sim \mathcal{N}(36,1)$ \\
\hline
\end{tabular}

TABLE 3: Optimal order quantities and reorder points.

\begin{tabular}{lcc}
\hline & $Q_{i}$ & $s_{i}$ \\
\hline Eggs & 2405.6 & 1288.0 \\
Dairy products & 3423.8 & 2288.0 \\
Grains & 5400.5 & 1288.0 \\
Meat products & 2436.9 & 1280.5 \\
\hline
\end{tabular}

$\widetilde{\widetilde{p}}_{i}$ (unit selling price of product $i$ ), $\widetilde{\widetilde{c}}_{i 1}$ (unit acquisition cost of product $i$ ), and $\widetilde{\widetilde{c}}_{i 2}$ (unit shortage cost of product $i$ ). These birandom variables are listed in Table 2 . The decision maker set confident levels $\alpha_{1}=\alpha_{2}=\alpha_{3}=\alpha_{4}=\beta=0.8$ and weighs $w_{1}=w_{2}=w_{3}=w_{4}=0.25$.

We implement the birandom simulation-based genetic algorithm in Matlab 7.0 (the population size $N_{\text {pop-size }}=30$, the probability of crossover $P_{c}=0.3$, and the probability of mutation $P_{m}=0.2$ ) and ran on a PC, $2.40 \mathrm{GHz}$ with $1024 \mathrm{MB}$ memory. After running 500 generations, the final solution is listed in Table 3. In addition, the objective function values are $\left(\bar{f}_{1}^{*}, \bar{f}_{2}^{*}, \bar{f}_{3}^{*}, \bar{f}_{4}^{*}\right)=(1878.8,1256.6,2890.3,3097.4)$.

\section{Further Discussions}

To show the effectiveness of the model and algorithm better, evaluation, analysis, and comparison from various aspects are presented.

5.1. Algorithm Evaluation. We evaluate the birandom simulation-based algorithm from two aspects.

First, we will show that the birandom simulation-based algorithm is robust to genetic parameters. We compare the results by the algorithm with different parameters, including population size $N_{\text {pop-size }}$, probability of crossover $P_{c}$, probability of mutation $P_{m}$, cycles in birandom simulation, and generations in GA. These results are listed in Table 4. It appears that almost all the objective values differ little from each other. In fact, each relative error does not exceed 3\% when different parameters are selected, which implies that the proposed algorithm is robust to the parameters setting and effective to solve the problem in this paper.

Second, since the functions in model (52) are linear, by Lemmas 13 and 14, model (52) can be transformed into its crisp equivalent model. Assume that all the parameters are the same and the final objective function values are $\left(\bar{f}_{1}^{*}, \bar{f}_{2}^{*}, \bar{f}_{3}^{*}, \bar{f}_{4}^{*}\right)=(1881.2,1253.6,2895.3,3093.8)$ by using the weighted sum method. The results obtained by the birandom simulation genetic algorithm and by the traditional weighted sum method are close to each other, which implies the effectiveness of the birandom simulation-based genetic algorithm proposed in this paper.

5.2. Sensitivity Analysis. However, under a different decision making environment and different conditions, the decision maker may alter the predetermined confidence levels. Then the optimal solution will alter accordingly. In order to show how the optimal solutions change along with the predetermined confidence levels, we conducted a sensitivity analysis as shown in Table 5. As shown from Table 5, a bigger $\beta$ induces a better result, because a bigger $\beta$ extends the feasible region. Conversely, a bigger $\alpha_{i}$ induces a worse result since a bigger $\alpha_{i}$ narrows the feasible region.

5.3. Model Comparison. Birandom system is a generation for stochastic system. For illustrating the advantage of birandom system, all the birandom variables in (52) are assumed to be random variables, and other parameters keep original values. Each birandom variable in (52) has the following form:

$$
\widetilde{\xi} \sim \mathcal{N}\left(\widetilde{\xi}, \sigma^{2}\right), \quad \tilde{\xi} \sim \mathcal{N}\left(\mu, \sigma^{\prime 2}\right) .
$$

Replace $\tilde{\xi}$ with $\mu$, which is a constant. Then $\widetilde{\widetilde{\xi}}$ degenerates into a random variable. For example, we use $\widetilde{p}_{11}(\sim \mathcal{N}(16,1))$ to replace $\widetilde{\widetilde{p}}_{11}$. As all the birandom variables are replaced by 
TABLE 4: Comparison of the results obtained with different parameters.

\begin{tabular}{lcccccc}
\hline & $N_{\text {pop-size }}$ & $P_{c}$ & $P_{m}$ & Cycles & Generations & $\left(\bar{f}_{1}^{*}, \bar{f}_{2}^{*}, \bar{f}_{3}^{*}, \bar{f}_{4}^{*}\right)$ \\
\hline 1 & 20 & 0.2 & 0.2 & 400 & 400 & $(1878.8,1256.6,2890.3,3097.4)$ \\
2 & 30 & 0.3 & 0.3 & 400 & 400 & $(1865.9,1259.8,2887.2,3104.5)$ \\
3 & 40 & 0.4 & 0.4 & 400 & 400 & $(1872.4,1257.6,2891.1,3099.2)$ \\
4 & 20 & 0.2 & 0.2 & 500 & 500 & $(1873.8,1257.2,2891.3,3099.4)$ \\
5 & 30 & 0.3 & 0.3 & 500 & 500 & $(1873.8,1256.6,2893.3,3098.6)$ \\
6 & 40 & 0.4 & 0.4 & 500 & 500 & $(1877.5,1256.8,2891.6,3099.7)$ \\
7 & 20 & 0.2 & 0.2 & 600 & 600 & $(1877.7,1257.7,2892.3,3098.5)$ \\
8 & 30 & 0.3 & 0.3 & 600 & 600 & $(1876.8,1259.6,2888.3,3099.4)$ \\
9 & 40 & 0.4 & 0.4 & 600 & 700 & $(1879.7,1259.6,2891.2,3099.5)$ \\
10 & 20 & 0.2 & 0.2 & 700 & 700 & $(1880.8,1257.6,2891.3,3096.4)$ \\
11 & 30 & 0.3 & 0.3 & 700 & 700 & $(1874.7,1256.5,2892.3,3093.5)$ \\
12 & 40 & 0.4 & 0.4 & 700 & $(1874.8,1254.6,2890.9,3097.2)$ \\
\hline
\end{tabular}

"Cycles" are the cycles in simulation. "Generations" are the generations in GA.

TABLE 5: Sensitivity analysis.

\begin{tabular}{lc}
\hline & $\left(\bar{f}_{1}^{*}, \bar{f}_{2}^{*}, \bar{f}_{3}^{*}, \bar{f}_{4}^{*}\right)$ \\
\hline$\alpha_{1}=\alpha_{2}=\alpha_{3}=\alpha_{4}=0.8, \beta=0.75$ & $(1873.8,1250.1,2878.2,3067.2)$ \\
$\alpha_{1}=\alpha_{2}=\alpha_{3}=\alpha_{4}=0.8, \beta=0.85$ & $(1892.5,1266.6,2896.7,3110.5)$ \\
$\alpha_{1}=\alpha_{2}=\alpha_{3}=\alpha_{4}=0.75, \beta=0.80$ & $(1885.8,1258.4,2882.1,3097.6)$ \\
$\alpha_{1}=\alpha_{2}=\alpha_{3}=\alpha_{4}=0.85, \beta=0.80$ & $(1866.8,1254.6,2885.3,3091.9)$ \\
\hline
\end{tabular}

random variables, equilibrium chance $\mathrm{Ch}^{e}$ degenerates into probability Pr. Then (52) can be written as

$$
\begin{array}{ll}
\max & \left\{\bar{f}_{1}, \bar{f}_{2}, \ldots, \bar{f}_{m}\right\} \\
\text { s.t. } & \operatorname{Pr}\left\{\widetilde{T C}_{i} \geq \bar{f}_{i}\right\} \geq \alpha_{i}, \quad i=1,2, \ldots, n, \\
& K_{i} Q_{i} \leq W_{i}, \quad i=1,2, \ldots, n, \\
& Q_{i} \geq s_{i}, \quad i=1,2, \ldots, n, \\
& \operatorname{Pr}\left\{\sum_{i=1}^{n}\left(A_{i}+\widetilde{C}_{i 1}+\widetilde{C}_{i 2}+\widetilde{C}_{i 3}\right) \leq B\right\} \leq \beta, \\
& Q_{i} \geq 0, \quad s_{i} \geq 0, \quad i=1,2, \ldots, n .
\end{array}
$$

This stochastic system is a special case of a birandom system, which can be solved using a stochastic simulation-based GA. Similar to the birandom simulation-based GA, the stochastic simulation-based GA is formed by embedding stochastic simulation into a GA. The robustness of the stochastic simulation-based GA can be verified as similar to the birandom simulation-based GA.

In order to make a comparison with the birandom system, we solve problem (54) with following parameter: $N_{\text {pop-size }}=30, P_{c}=0.3$, and $P_{m}=0.2,500$ cycles in stochastic simulation, 500 generations in GA. The final result is $\left(\bar{f}_{1}^{*}, \bar{f}_{2}^{*}, \bar{f}_{3}^{*}, \bar{f}_{4}^{*}\right)=(1873.1,1231.5,2790.2,3016.9)$, which is worse than the result of the birandom system. Thus it shows that birandom system is more flexible and can generate better objective values than the corresponding stochastic system.
5.4. Managerial Insight. Some managerial insights can be derived from the above discussions. From the sensitivity analysis, we can see the following. (1) A pessimistic decision maker will set lower confidence levels to avoid higher risk. As a result, the values for the objective functions are worse than those at higher confidence levels. (2) An optimistic decision maker will set higher confidence levels to obtain better values for the objective functions. However, there will be the cost of higher risk. To sum up, the values for the objective functions depend on the attitude of the decision maker towards risk. From a comparison between the stochastic model and the birandom model, the decision maker can obtain more flexible results if they can construct a birandom model rather than a stochastic model in a complex stochastic decision environment. However, depicting the complex stochastic decision environment as birandom variables costs more, so the decision maker must make a trade-off between the cost and the benefit.

\section{Conclusions}

In this paper, we have formulated an equilibrium chanceconstrained multiobjective programming model with birandom parameters, which extends the general multiobjective programming model. We converted a special linear model into a crisp multiobjective programming model which can be solved using traditional techniques. A modified genetic algorithm was designed by embedding the birandom simulation technique to deal with the general model. By assuming that some parameters (unit selling price, unit acquisition cost, etc.) are birandom variables, we developed an equilibrium chance-constrained multiobjective programming model with birandom parameters for an inventory problem and solved it using the birandom simulation-based GA. Further discussions show the effectiveness of the proposed model and algorithm. Some managerial insights may help decision makers improve their decision making.

Though the inventory problem considered is specific, the theoretical results can be extended to various inventory problems. In the future, detailed analysis, further research, and 
practical application of the model and algorithm proposed in this paper will be considered.

\section{Acknowledgments}

The authors would like to give their great appreciates to the editors and anonymous referees for their helpful comments and valuable suggestions which improved the content and composition substantially. This research was supported by the Programs of NSFC (Grant nos. 70831005 and 71273036), Projects of International Cooperation and Exchanges NSFC (Grant no. 71011140076), “985” Program of Sichuan University (Innovative Research Base for Economic Development and Management), Sichuan University Young Teachers Scientific Research Start Funds (Grant no. 2012SCU11016), and the Key Program of Sichuan System Science and Enterprise Development Research Center (Grant no. Xq13B04).

\section{References}

[1] S. Graves, A Single-Item Inventory Model for a Non-stationary demand Process, Sloan School of Management, Massachusetts Institute of Technology, 1997.

[2] M. Khouja and S. Goyal, "Single item optimal lot sizing under continuous unit cost decrease," International Journal of Production Economics, vol. 102, no. 1, pp. 87-94, 2006.

[3] J. Riezebos and G. Gaalman, "A single-item inventory model for expected inventory order crossovers," International Journal of Production Economics, vol. 121, no. 2, pp. 601-609, 2009.

[4] M. Hariga, "A single-item continuous review inventory problem with space restriction," International Journal of Production Economics, vol. 128, no. 1, pp. 153-158, 2010.

[5] G. Kiesmüller, A. De Kok, and S. Dabia, "Single item inventory control under periodic review and a minimum order quantity," International Journal of Production Economics, vol. 133, no. 1, pp. 280-285, 2011.

[6] D. K. Bhattacharya, "On multi-item inventory," European Journal of Operational Research, vol. 162, no. 3, pp. 786-791, 2005.

[7] A. Roy, S. Kar, and M. Maiti, "A deteriorating multi-item inventory model with fuzzy costs and resources based on two dierent defuzzification techniques," Applied Mathematical Modelling, vol. 32, no. 2, pp. 208-223, 2008.

[8] U. K. Bera, M. Rong, N. K. Mahapatra, and M. Maiti, "A multiitem mixture inventory model involving random lead time and demand with budget constraint and surprise function," Applied Mathematical Modelling, vol. 33, no. 12, pp. 4337-4344, 2009.

[9] P. Guchhait, M. Maiti, and M. Maiti, "Multi-item inventory model of breakable items with stock-dependent demand under stock and time dependent breakability rate," Computers \& Industrial Engineering, vol. 59, no. 4, pp. 911-920, 2010.

[10] H. Baker and R. Ehrhardt, "A dynamic inventory model with random replenishment quantities," Omega, vol. 23, no. 1, pp. 109-116, 1995.

[11] L. Wang, Q. L. Fu, C. G. Lee, and Y. R. Zeng, "Model and algorithm of fuzzy joint replenishment problem under credibility measure on fuzzy goal," Knowledge-Based Systems, vol. 39, pp. 57-66, 2013.

[12] A. Roy, M. K. Maiti, S. Kar, and M. Maiti, "An inventory model for a deteriorating item with displayed stock dependent demand under fuzzy inflation and time discounting over a random planning horizon," Applied Mathematical Modelling, vol. 33, no. 2, pp. 744-759, 2009.

[13] M. K. Maiti and M. Maiti, "Fuzzy inventory model with two warehouses under possibility constraints," Fuzzy Sets and Systems, vol. 157, no. 1, pp. 52-73, 2006.

[14] P. Dutta, D. Chakraborty, and A. R. Roy, "A single-period inventory model with fuzzy random variable demand," Mathematical and Computer Modelling, vol. 41, no. 8-9, pp. 915-922, 2005.

[15] J. Xu and L. Zhao, "A class of fuzzy rough expected value multiobjective decision making model and its application to inventory problems," Computers \& Mathematics with Applications, vol. 56, no. 8, pp. 2107-2119, 2008.

[16] J. Xu and L. Zhao, "A multi-objective decision-making model with fuzzy rough coefficients and its application to the inventory problem," Information Sciences, vol. 180, no. 5, pp. 679-696, 2010.

[17] A. Charnes and W. W. Cooper, "Chance-constrained programming," Management Science, vol. 6, pp. 73-79, 1959.

[18] A. Geletu, M. Klöppel, H. Zhang, and P. Li, "Advances and applications of chance-constrained approaches to systems optimisation under uncertainty," International Journal of Systems Science, vol. 44, no. 7, pp. 1209-1232, 2013.

[19] J. Peng and B. Liu, "Birandom variables and birandom programming," Computers \& Industrial Engineering, vol. 53, pp. 433-453, 2007.

[20] Y.-K. Liu and B. Liu, "Fuzzy random programming with equilibrium chance constraints," Information Sciences, vol. 170, no. 2-4, pp. 363-395, 2005.

[21] J. Xu and Z. Tao, "A class of multi-objective equilibrium chance maximization model with twofold random phenomenon and its application to hydropower station operation," Mathematics and Computers in Simulation, vol. 85, pp. 11-33, 2012.

[22] G. Zhou and M. Gen, "Genetic algorithm approach on multicriteria minimum spanning tree problem," European Journal of Operational Research, vol. 114, no. 1, pp. 141-152, 1999.

[23] M. Gen and R. Cheng, Genetic Algorithms and Engineering Optimization, Wiley-interscience, 2000.

[24] M. Soolaki, I. Mahdavi, N. Mahdavi-Amiri, R. Hassanzadeh, and A. Aghajani, "A new linear programming approach and genetic algorithm for solving airline boarding problem," Applied Mathematical Modelling, vol. 36, no. 9, pp. 4060-4072, 2012.

[25] J. Rezaei and M. Davoodi, "A deterministic, multi-item inventory model with supplier selection and imperfect quality," Applied Mathematical Modelling, vol. 32, no. 10, pp. 2106-2116, 2008.

[26] H. Nazif and L. S. Lee, "Optimised crossover genetic algorithm for capacitated vehicle routing problem," Applied Mathematical Modelling, vol. 36, no. 5, pp. 2110-2117, 2012. 


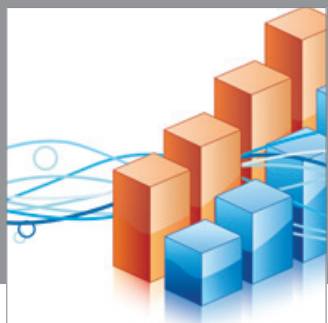

Advances in

Operations Research

mansans

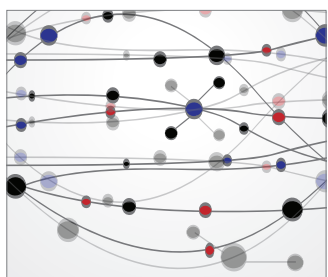

The Scientific World Journal
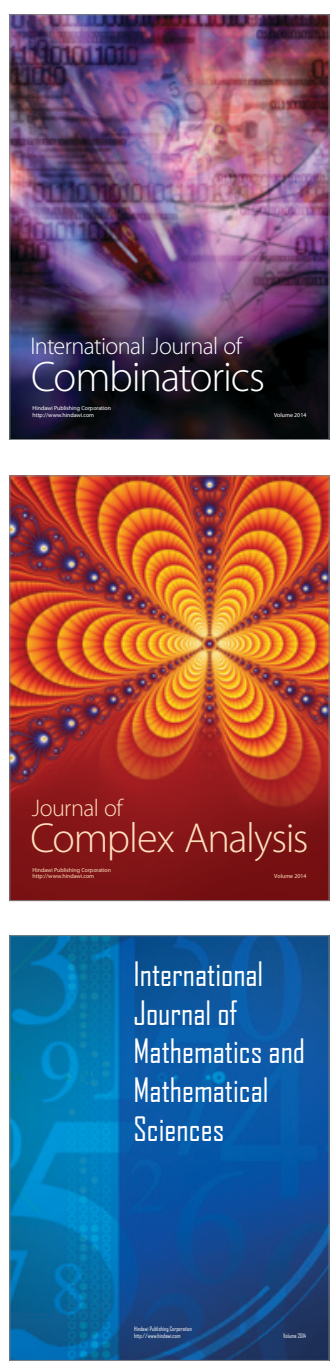
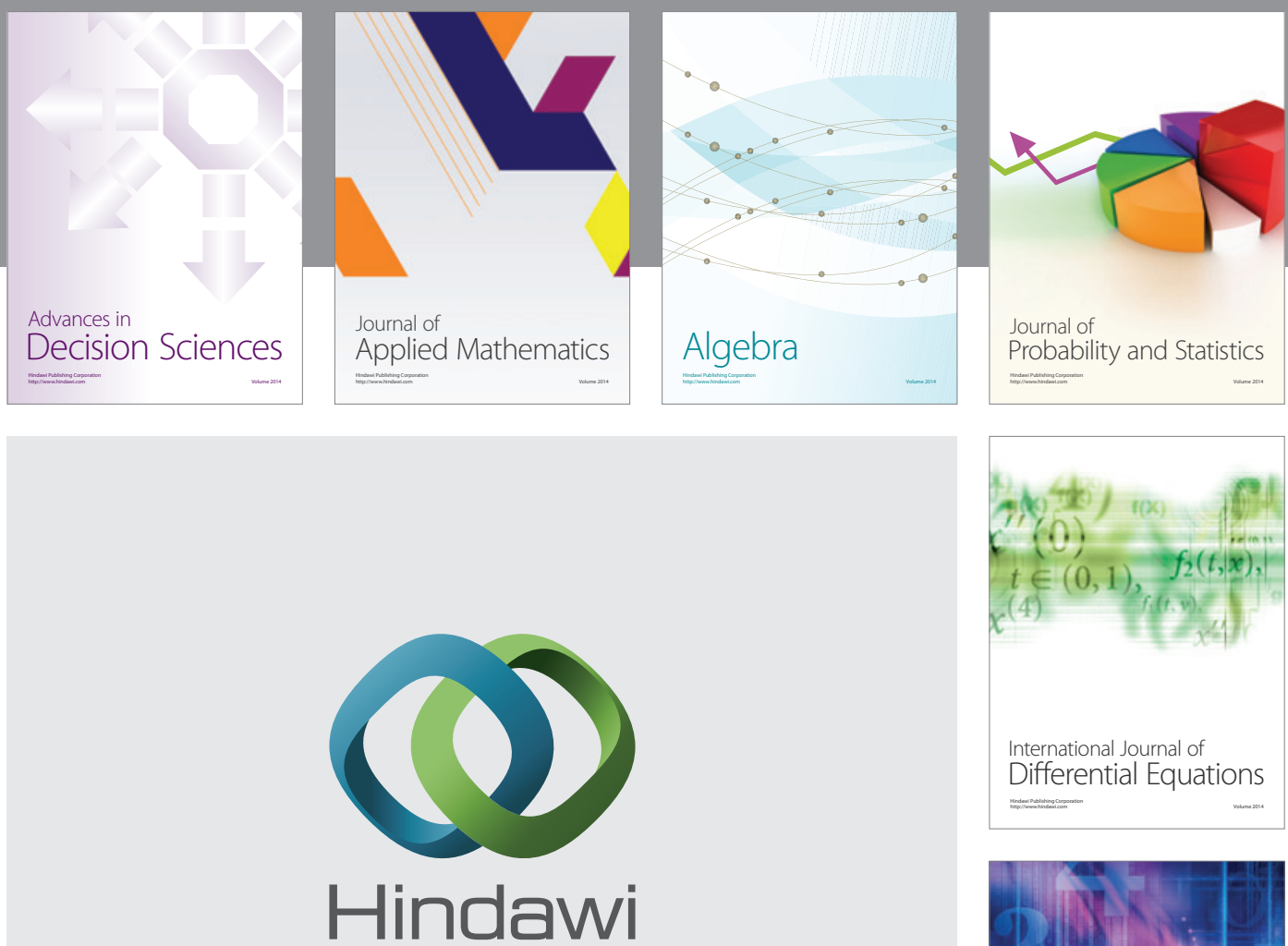

Submit your manuscripts at http://www.hindawi.com
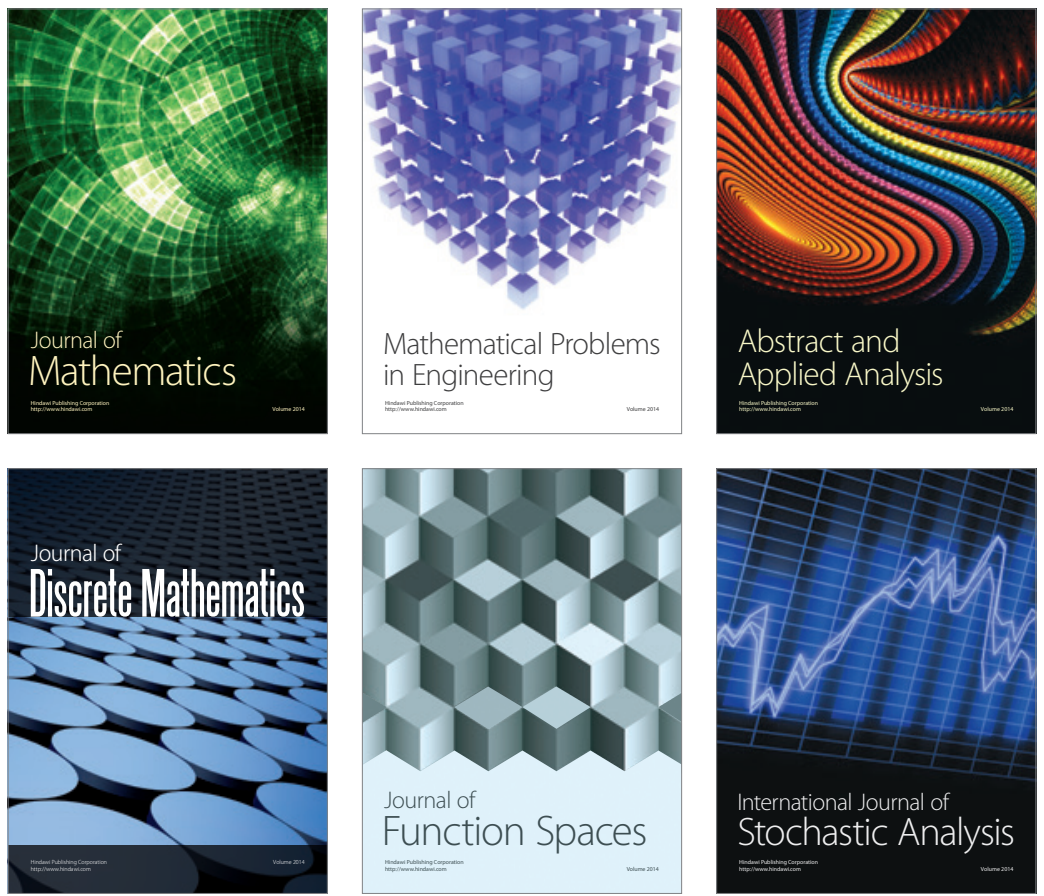

Journal of

Function Spaces

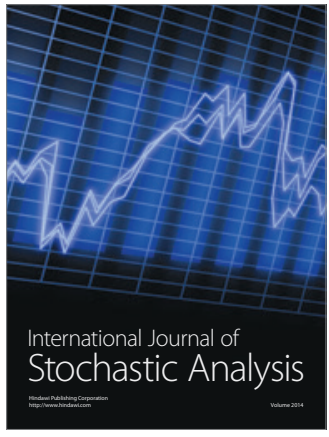

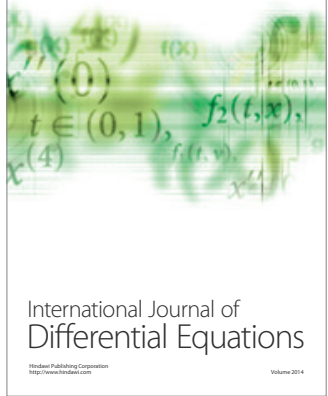
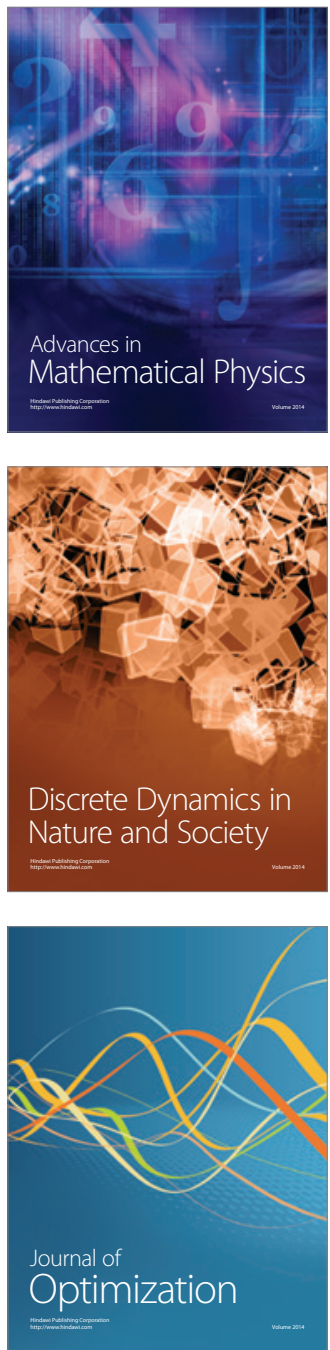